\title{
Energy and Hole Transfer between Dyes Attached to Titania in Cosensitized Dye-Sensitized Solar Cells
}

\author{
Brian E. Hardin, ${ }^{\dagger}$ Alan Sellinger, ${ }^{\dagger}$ Thomas Moehl, ${ }^{\ddagger}$ Robin Humphry-Baker, ${ }^{\ddagger}$ Jacques-E. Moser, ${ }^{\ddagger}$ Peng Wang, ${ }^{\S}$ \\ Shaik M. Zakeeruddin, ${ }^{\neq}$Michael Grätzel, ${ }^{*, \neq}$ and Michael D. McGehee ${ }^{*,+}$ \\ ${ }^{\dagger}$ Department of Materials Science and Engineering, Stanford University, Stanford, California 94305, United States \\ ${ }^{\ddagger}$ Laboratoire de Photonique et Interfaces, École Polytechnique Fédérale de Lausanne, Switzerland \\ ${ }^{\S}$ Changchun Institute of Applied Chemistry, Chinese Academy of Sciences, Changchun 130022, People’s Republic of China
}

Supporting Information

\begin{abstract}
Cosensitization of broadly absorbing ruthenium metal complex dyes with highly absorptive near-infrared (NIR) organic dyes is a clear pathway to increase near-infrared light harvesting in liquidbased dye-sensitized solar cells (DSCs). In cosensitized DSCs, dyes are intimately mixed, and intermolecular charge and energy transfer processes play an important role in device performance. Here, we demonstrate that an organic NIR dye incapable of hole regeneration is able to produce photocurrent via intermolecular energy transfer with an average excitation transfer efficiency of over $25 \%$ when cosensitized with a metal complex sensitizing dye (SD). We also show that intermolecular hole transfer from the SD to NIR dye is a competitive

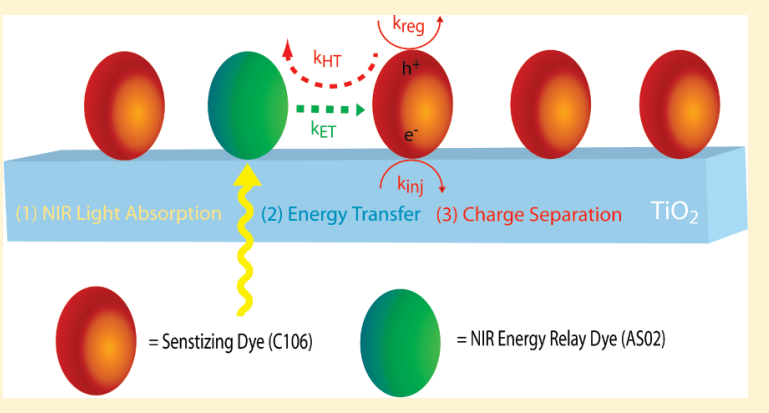
process with dye regeneration, reducing the internal quantum efficiency and the electron lifetime of the DSC. This work demonstrates the general feasibility of using energy transfer to boost light harvesting from 700 to 800 nm and also highlights a key challenge for developing highly efficient cosensitized dye-sensitized solar cells.
\end{abstract}

\section{INTRODUCTION}

Dye-sensitized solar cells comprised mainly of abundant, nontoxic materials offer an inexpensive route to develop highly efficient photovoltaic cells. ${ }^{1-4}$ Currently, the most efficient sensitizing dyes are ruthenium-based, metal ligand complexes (e.g., C106 and N719), 5,6 which absorb light in the visible portion of the solar spectrum, have excellent charge injection properties, and produce a high open-circuit voltage, $V_{\mathrm{oc}}$, which is defined as greater than $750 \mathrm{mV}$. It should be possible to further increase the power conversion efficiency of DSCs by harvesting light in the near-infrared red portion of the spectrum. Cosensitization of titania by dyes with complementary absorption spectra has been demonstrated to broaden the spectral response of organic dye-based DSCs in the visible portion of the spectrum, but not beyond $720 \mathrm{~nm} \cdot{ }^{7-10}$ Designing near-infrared sensitizing dyes with high internal quantum efficiencies is challenging because reducing the band gap requires more precise alignment of the LUMO and HOMO levels and short conjugated ligands to facilitate charge transfer. To date, only two NIR sensitizing dyes (i.e., peak absorption $>700 \mathrm{~nm}$ ) have demonstrated good charge injection efficiencies in DSCs, but neither dye has a $V_{\text {oc }}$ greater than $450 \mathrm{mV} .^{11,12}$ Recombination from the electrons in titania with holes in the dye and triiodide in the electrolyte plays a key role in determining the open-circuit voltage. ${ }^{13}$ Organic dyes typically experience higher recombination rates resulting in a lower $V_{\mathrm{oc} \cdot}{ }^{14}$ The great challenge of designing a cosensitized DSC system using NIR dyes will be maintaining a $V_{\text {oc }}$ greater than $700 \mathrm{mV}$.

Two NIR dye design strategies could result in higher power conversion efficiencies. First, it may be possible to use highly absorptive NIR-sensitizing dyes that directly inject charges even if NIR-SDs have higher recombination rates by using low surface concentrations $(<10 \%)$ of NIR-SDs to minimize $V_{\text {oc }}$ losses. DSC systems where cosensitized dyes do not electronically interact with one another are expected to have an electron recombination rate equivalent to the weighted average of the individual dye DSC systems. However, intermolecular charge transfer from dyes with a low recombination rate to dyes with a higher recombination rate can significantly increase the overall electron recombination rate between oxidized dyes and electrons in the titania, which can disproportionately reduce the open-circuit voltage of the cosensitized DSC system.

A second strategy is to electronically insulate the NIR dye from the $\mathrm{TiO}_{2}$ surface to reduce the recombination rate, which would maintain the $V_{\text {oc }}$ but also prevent electron injection. In this case, the NIR dye would act as an energy relay dye (ERD) requiring efficient intermolecular energy transfer to the metal complex SD to generate photocurrent, as shown in Scheme 1. To

\footnotetext{
Received: December 17, 2010

Revised: $\quad$ May 7, 2011

Published: May 27, 2011
} 
Scheme 1. NIR Dye Attached to the Titania Surface Absorbs Near-Infrared Photons and Uses Short-Range Energy

Transfer To Excite a Neighboring Sensitizing Dye, Which Is Responsible for Electron Transfer into the $\mathrm{TiO}_{2}\left(k_{\mathrm{inj}}\right)$ and Hole Regeneration with the Electrolyte $\left(k_{\text {reg }}\right)$

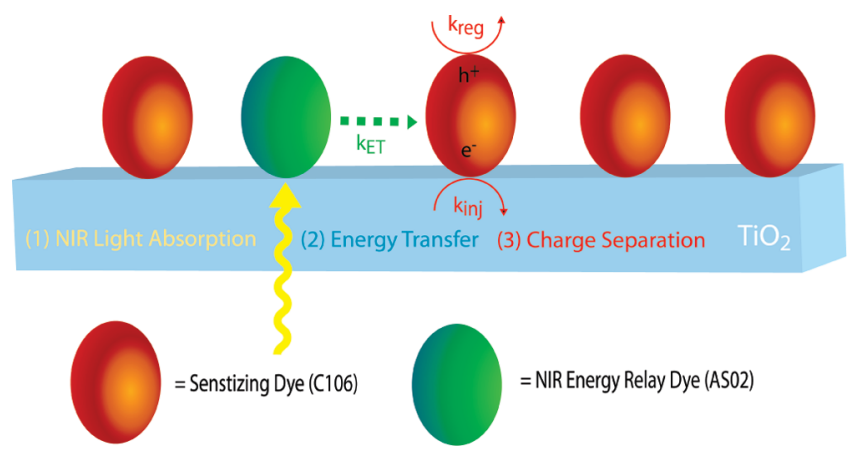

address the feasibility of using NIR-ERDs, we must first determine how effectively NIR-ERDs can transfer energy to SDs in a cosensitized system.

Conventional DSCs are solely dependent on charge transfer mechanisms for current generation, while plants often incorporate a variety of energy transfer processes to increase light harvesting during photosynthesis. ${ }^{15}$ Developing systems that incorporate both Förster resonant energy transfer (FRET) ${ }^{16}$ and Dexter ${ }^{17}$ energy transfer allow greater flexibility in the design of potential light harvesting candidates. Energy relay dyes (ERDs) have been used previously to increase light harvesting in the blue portion of the solar spectrum. ${ }^{18,19}$

Blue ERDs, which absorb high energy photons and undergo FRET to sensitizing dyes, can efficiently transfer energy when placed inside the electrolyte ${ }^{18,20}$ or cosensitized ${ }^{21}$ on nanocrystalline $\mathrm{TiO}_{2}$. Grimes et al. recently demonstrated that ERDs unattached to the titania and slightly red-shifted relative to the sensitizing dye peak absorption were able to undergo FRET to the SD. ${ }^{22}$ However, the low FRET radii (e.g., $1-4 \mathrm{~nm}$ ) due to the poor overlap between ERD emission and SD absorption prevents efficient energy transfer from occurring when ERDs are placed inside the electrolyte. ${ }^{23}$ For DSC systems where energy transfer is weak (i.e., FRET radii $<4 \mathrm{~nm}$ ), NIR-ERDs should be within the FRET radius of the SD to efficiently transfer energy, which requires tethering between dyes ${ }^{19}$ or cosensitization on the $\mathrm{TiO}_{2}$ surface. ${ }^{21}$

\section{RESULTS AND DISCUSSION}

2.1. Charge Transfer Rates and Energy Transfer Estimates in the Cosensitized C106/AS02 System. To verify that energy transfer occurs from the NIR dye to the SD, we have designed a zinc naphthalocyanine-based dye (AS02) that cannot regenerate with the electrolyte and produce photocurrent independently. The absorption, emission, and the chemical structure of $\mathrm{C} 106$ and AS02 in dimethylformamide (DMF) are shown in Figure 1. C106 has a peak molar extinction coefficient of $18700 \mathrm{M}^{-1} \mathrm{~cm}^{-1}$ at $550 \mathrm{~nm}$ with an absorption tail that extends weakly out to $800 \mathrm{~nm} .{ }^{5} \mathrm{C} 106$ has a broad emission spectrum with a peak at 786 and a natural fluorescence decay lifetime of 85 ns in DMF (see Supporting Information S5). The photoluminescence quantum efficiency of Ru-based metal complexes is between $0.2 \%$ and $0.02 \% .{ }^{24} \mathrm{ASO} 2$ has a peak molar extinction coefficient of $100000 \mathrm{M}^{-1} \mathrm{~cm}^{-1}$ at

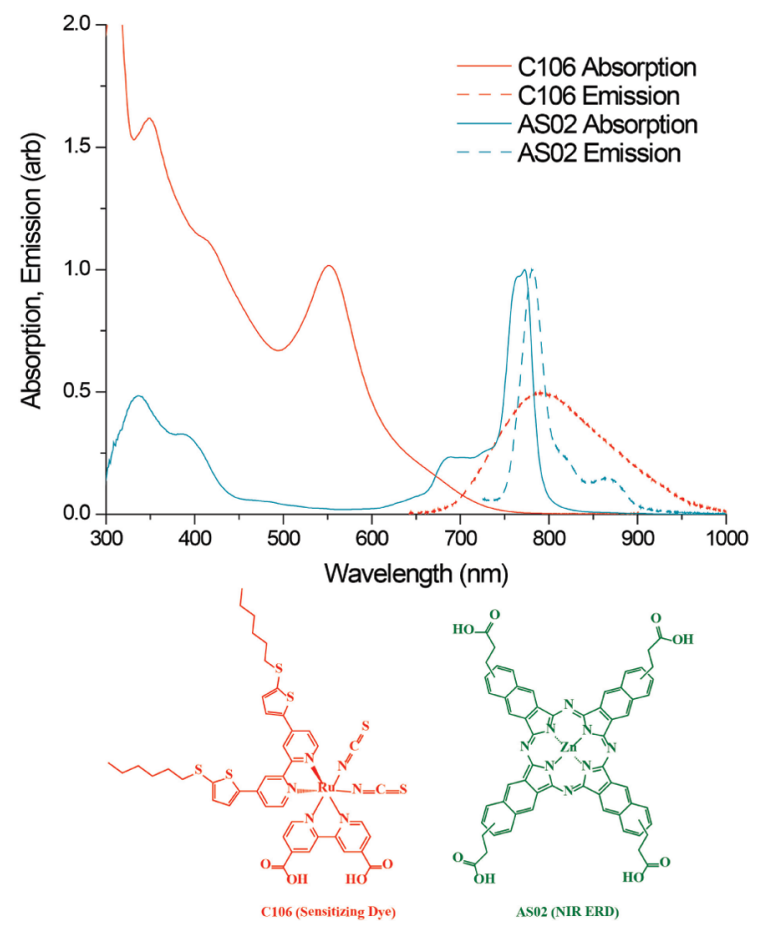

Figure 1. Absorption and emission spectra of the sensitizing dye, C106, and near-infrared dye, AS02, in DMF. The chemical structures of C106 and AS02 are shown in the inset.

$773 \mathrm{~nm}$ with a narrow emission peak at $782 \mathrm{~nm}$ with a fluorescence natural decay lifetime of $2.75 \mathrm{~ns}$ in DMF. The photoluminescence quantum efficiency of Zn-based naphthalocyanines is between $10 \%$ and $30 \% .{ }^{25}$ Photoelectron spectroscopy in air (PESA) was used to determine that the HOMO level of AS02 $(-4.60 \mathrm{eV})$ is high relative to the iodide potential $(-4.85$ $\mathrm{eV}$ ), which has previously been shown to prevent dye regeneration for a similar $\mathrm{Zn}$-based naphthalocyanine sensitizing dye; ${ }^{26}$ $\mathrm{C} 106$ has a HOMO level of $-5.27 \mathrm{eV}{ }^{5}$ Intermolecular hole transfer is thermodynamically favorable from the C106 to the AS02; the rate of transfer will be dependent upon the HOMO level offset and the separation distance between molecules.

The Förster radius $\left(R_{0}\right)$ is the distance between the donor and acceptor dye when Förster resonant energy transfer is 50\% likely. The FRET $R_{0}$ from the donor to the acceptor dye is primarily determined by the donor photoluminescence quantum efficiency, the molar extinction coefficient of the acceptor, and the overlap between the donor emission and acceptor absorption spectra (see the Supporting Information). Traditional energy transfer systems are designed to funnel energy from a donor chromophore whose absorption is blue-shifted relative to the acceptor dye absorption (i.e., C106 to AS02) so that donor emission can overlap with the peak acceptor absorption to provide the largest possible FRET radius. ${ }^{27}$ The FRET radius from C106 to AS02 is estimated to be between 1.5 and $2.2 \mathrm{~nm}$, which is fairly short and primarily due to the low photoluminescence quantum efficiency of the C106 dye. Despite the weak emission/absorption overlap of the AS02 emission and C106 absorption, the FRET radius from the NIR dye (AS02) to the SD (C106) is estimated to be between 1.5 and $1.8 \mathrm{~nm}$. The rate of Förster energy transfer $\left(k_{\mathrm{FRET}}\right)$ between isolated chromophores, known as point-to-point transfer, is given by $k_{\mathrm{FRET}}=k_{0}\left(R_{0}\right)^{6} / r^{6}$, where $r$ is the separation distance and $k_{0}$ is the natural 
Scheme 2. Jablonski Plot of AS02 + C106 DSC System ${ }^{a}$

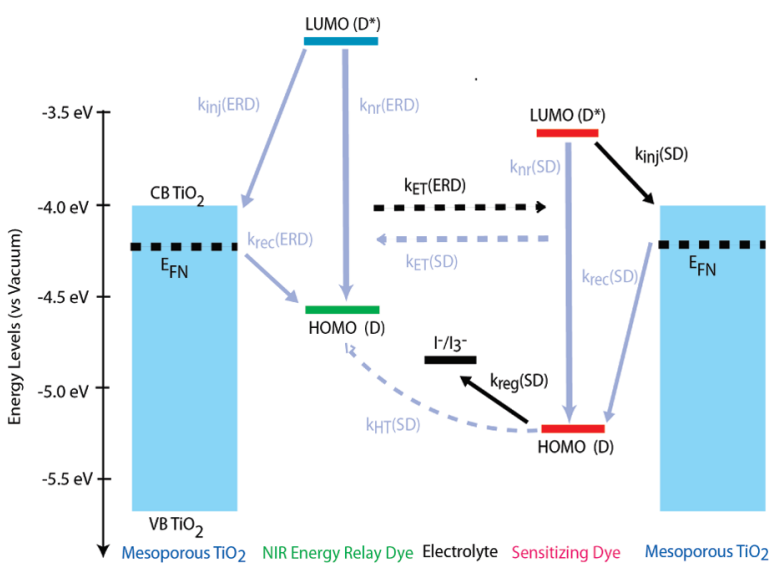

${ }^{a}$ The scheme is not geometrically correct (i.e., both dyes should be on the same $\mathrm{TiO}_{2}$ surface); processes that result in photocurrent generation are labeled in black, while processes that do not contribute to photocurrent are labeled in grey; dashed lines represent intermolecular processes.

fluorescence decay rate, $k_{0}=1 / \tau_{0}$. The separation distance can be approximated on the basis of the sensitizing dye surface concentration, which was measured by desorbing the C106 from titania using $0.15 \mathrm{M}$ tetrabutylammonium hydroxide in DMF and found to be 1 dye $/ \mathrm{nm}^{2}$ on the $17 \mathrm{~nm}$ diamter $\mathrm{TiO}_{2}$ nanoparticles with an estimated roughness factor of $100 / \mu \mathrm{m}$ (see the Supporting Information). When the NIR dye molecules intimately mix with the C106, the average separation between dyes is estimated to be approximately $1 \mathrm{~nm}$. The FRET rate from the AS02 to C106 is predicted to be between $7.1 \times 10^{9}$ and $2.3 \times$ $10^{10} \mathrm{~s}^{-1}\left(\tau_{\text {FRET,ASO2 }}=44-130 \mathrm{ps}\right)$ based on an average separation distance of $1 \mathrm{~nm}$, while the FRET rate is estimated from C106 to AS02 to be between $1.3 \times 10^{8}$ and $1.3 \times 10^{9} \mathrm{~s}^{-1}$ $\left(\tau_{\text {FRET,C106 }}=0.75-7.5 \mathrm{~ns}\right)$. Interestingly, the FRET rate from the NIR dye (AS02) to the visible sensitizing dye (C106) is an order of magnitude faster than in the opposite direction due to the differences in the fluorescence decay rates between chromophores. The $k_{\mathrm{FRET}}$ rates should be considered rough approximations because the FRET radius calculation is based on a random orientation (i.e., dyes rotating freely in solution), which would not be the case when anchored on the $\mathrm{TiO}_{2}$ surface. Given the short length scale, Dexter energy transfer may also play an important role in intermolecular energy transfer. ${ }^{27}$ Meyer et al. have demonstrated near unity lateral Dexter energy transfer from Ru-based metal complex SDs to Os-based metal complex SDs across a semiconductor interface ${ }^{28}$ and have also estimated Dexter energy transfer rates between Ru metal complex SDs to be on the 30 ns time scale. ${ }^{29}$ Calculating the Dexter transfer rate between AS02 to $\mathrm{C} 106$ requires calculating the inner and outer sphere reorganization energies and is beyond the scope of this work. ${ }^{29}$

2.2. Excitation Transfer Efficiency Calculations. The excitation transfer efficiency, ETE, is the probability that a dye will undergo energy transfer. ETE is determined by the rate of intermolecular energy transfer $\left(k_{\mathrm{ET}}\right)$ relative to the combined rates of all decay pathways, which includes the electron injection rate $\left(k_{\text {inj }}\right)$ and the nonradiative decay rate $\left(k_{\mathrm{nr}}\right)$ of the attached dye as shown in eq 1 . Hole regeneration is an alternative decay pathway, but occurs on time scales several orders of magnitude
Table 1. Energy and Charge Transfer Lifetimes for AS02 and C106

\begin{tabular}{|c|c|c|c|}
\hline mechanism & name & $\begin{array}{l}\text { ERD } \\
\text { lifetime }\end{array}$ & SD lifetime \\
\hline $\mathrm{e}^{-}$injection into $\mathrm{TiO}_{2}$ & $k_{\text {inj }}$ & $\leq 230 \mathrm{ps}$ & $0.02-100 \mathrm{ps}^{a}$ \\
\hline $\mathrm{h}^{+}$regeneration with electrolyte & $k_{\text {reg }}$ & & $3.6 \mu \mathrm{s}$ \\
\hline nonradiative recombination & $k_{\mathrm{nr}}$ & $1.5 \mathrm{~ns}$ & $18.5 \mathrm{~ns}$ \\
\hline $\begin{array}{l}\mathrm{e}^{-}\left(\mathrm{TiO}_{2}\right) \text { recombination } \\
\text { with } \mathrm{h}^{+}(\text {dye })\end{array}$ & $k_{\mathrm{rec}}$ & $8.0 \mathrm{~ms}^{b}$ & $590 \mu \mathrm{s}$ \\
\hline intermolecular $\mathrm{h}^{+}$transfer & $k_{\mathrm{HT}}$ & & $\leq 5.4 \mu \mathrm{s}$ \\
\hline $\begin{array}{l}\text { natural fluorescence decay } \\
\text { in DMF }\end{array}$ & $k_{0}$ & $2.75 \mathrm{~ns}$ & $85 \mathrm{~ns}$ \\
\hline modeled intermolecular FRET & $k_{\text {FRET }}$ & $44-130 \mathrm{ps}$ & $0.75-7.5 \mathrm{~ns}$ \\
\hline measured intermolecular ET & $k_{\mathrm{ET}}$ & $\leq 530 \mathrm{ps}$ & \\
\hline \multicolumn{4}{|c|}{$\begin{array}{l}{ }^{a} \text { Rates measured by Grätzel et al. }{ }^{30 b} \text { Rates measured by Durrant et al. }{ }^{26} \\
\text { The modeled } k_{\mathrm{ET}} \text { and } k_{\mathrm{HT}} \text { were based on measured rates and the ETE } \\
\text { and IQE, respectively. }\end{array}$} \\
\hline
\end{tabular}

slower than energy and electron transfer and is not a major factor for iodide/triiodide-based DSCs.

$$
\mathrm{ETE}=\frac{k_{\mathrm{ET}}}{k_{\mathrm{ET}}+k_{\mathrm{inj}}+k_{\mathrm{nr}}}
$$

The rates of the AS02 + C106 DSC system are shown in Scheme 2 with the rate lifetimes displayed in Table 1. Timeresolved PL measurements were performed on titania and alumina films to determine electron transfer to $\mathrm{TiO}_{2}\left(k_{\text {inj }}\right)$ and the nonradiative decay rates $\left(k_{\mathrm{nr}}\right)$, respectively. For efficient sensitizing dyes, the electron injection rate is the fastest kinetic process; the $k_{\text {inj }}$ rate of ASO2 is greater than $4.3 \times 10^{9} \mathrm{~s}^{-1}\left(\tau_{\text {inj,ASO2 }}<\right.$ $230 \mathrm{ps}$ ), while the $k_{\text {inj }}$ rate of Ru-based metal ligand-based DSCs is approximately $5 \times 10^{13} \mathrm{~s}^{-1}\left(\tau_{\text {inj,C106 }} \approx 20 \mathrm{fs}\right){ }^{30}$ It should be noted that the nonradiative decay rate of both dyes is faster when attached on alumina than the fluorescence decay rate when in DMF. Transient absorption decay measurements on dyed $\mathrm{TiO}_{2}$ films were used with and without the iodide-based electrolyte to determine the regeneration rate $\left(k_{\text {reg }}\right)$ between holes in the dye with the electrolyte and the recombination rate $\left(k_{\mathrm{rec}}\right)$ between holes in the dye and electrons in the titania respectively. All rates were best fit as a single exponential decay; the experimental details and data are provided in the Supporting Information.

The excitation transfer efficiency from NIR dye to the SD is estimated to be between $60 \%$ and $80 \%$ on the basis of the charge kinetics of the AS02 the FRET radius and an average separation distance of $1 \mathrm{~nm}$. DSCs cosensitized with all organic dyes have previously demonstrated an energy cascade effect, where intermolecular energy transfer occurs from the high band gap to the lower band gap SD, ${ }^{31}$ However, energy transfer from the metal complex SD to the NIR dye is not likely because the rate of electron injection of C106 is several orders of magnitude faster than energy transfer processes, efficiently splitting the exciton before energy transfer can occur.

2.3. Intermolecular Energy Transfer from AS02 to C106. To verify that intermolecular energy and hole transfer occurs in this DSC system, we cosensitized transparent $6.5 \mu \mathrm{m}$ thick $\mathrm{TiO}_{2}$ mesoporous films and measured the optical and electrical properties using methods similar to those in the literature. ${ }^{20}$ Showa Denko $17 \mathrm{~nm}$ diameter $\mathrm{TiO}_{2}$ particles were deposited on 

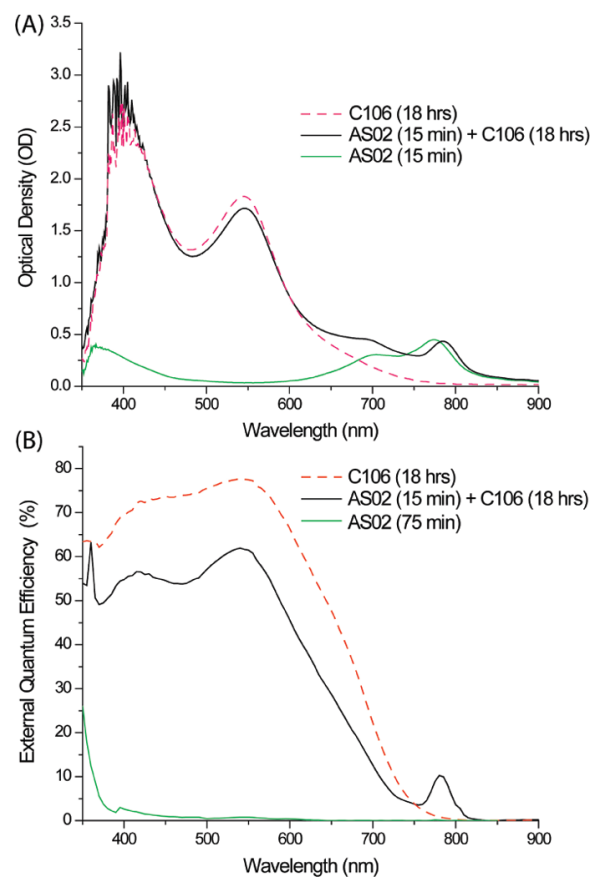

Figure 2. (A) Optical density versus wavelength of titania films sensitized with C106, AS02 + C106, and AS02 only. (B) External quantum efficiency versus wavelength of C106, ASO2 + C106, and AS02 only dye-sensitized solar cells.

fluorine-doped tin oxide glass (TEC $15 \Omega$ /square, $2.2 \mathrm{~mm}$ thick, Pilkington) via screen printing, sintered at $450{ }^{\circ} \mathrm{C}$, and subsequently treated with $\mathrm{TiCl}_{4}{ }^{32}$ Figure $2 \mathrm{~A}$ shows the optical density (OD) versus wavelength during different stages of cosensitization. The titania films were first dipped in a $0.1 \mathrm{mM}$ ASO2 solution in DMF for $15 \mathrm{~min}$, then rinsed in DMF and dried with $\mathrm{N}_{2}$ (green line). The film was subsequently dipped in a $0.3 \mathrm{mM}$ C106 solution comprised of 10\% DMF with $90 \%$ acetonitrile: tert-butyl alcohol (50:50 mixture by volume) for $18 \mathrm{~h}$ and rinsed in acetonitrile (black line). The control DSCs were dipped in the C106 solution for $18 \mathrm{~h}$ (red dashed lines). $\mathrm{TiO}_{2}$ films dipped in AS02 for 15 min resulted in fractional surface coverage of $14 \%$ AS02 (see the Supporting Information) with a peak optical density of 0.45 or $65 \%$ of light absorbed at $780 \mathrm{~nm}$. Adding the AS02 prior to C106 sensitization does not drastically affect the overall light harvesting of the C106 sensitizer. The peak OD of the $\mathrm{C} 106$ control device is 1.83 ( $98.5 \%$ light absorption) versus 1.74 (98.2\% light absorption) at $550 \mathrm{~nm}$ for the AS02 (14\%) + C106 (86\%) system. Figure 2A also shows a slight red shifting of both the AS02 and a C106 peak, which is likely caused by molecular orbital overlap between NIR dye. The red shift was not caused by solvatochromatic effects; changing from DMF to acetonitrile:tert-butyl alcohol mixture resulted in a slight blue shift in the absorption peak of the ASO2 sensitized on $\mathrm{TiO}_{2}$. The AS02 peak shape and intensity do not change during sequential sensitization, which indicates that the ASO2 molecules do not aggregate or desorb while being dipped in the C106 solution.

Dye-sensitized solar cells were assembled and tested using standard methods previously described in detail in the literature with an electrolyte comprised of $1.0 \mathrm{M}$ 1,3-dimethylimidazolium iodide, $0.03 \mathrm{M}$ iodide, $0.1 \mathrm{M}$ guanidinium thiocyante, and $0.5 \mathrm{M}$ tert-butylpyridine in acetonitrile valoronitrile $(85: 15 \mathrm{v} / \mathrm{v}))^{5,33}$ External quantum efficiency (EQE) measurements were used to verify intermolecular energy and hole transfer. The EQE at $780 \mathrm{~nm}$ is $10.2 \%$ for AS02 + C106 DSC and $0.8 \%$ for the $\mathrm{C} 106$ control as shown in Figure 2B. The EQE contribution from $\mathrm{ASO2}$ is the direct result of energy transfer from the AS02 to the C106. The EQE of ASO2-only DSCs (green line) showed no photoresponse at $780 \mathrm{~nm}$; the EQE generated below $450 \mathrm{~nm}$ is a result of light absorption by the titania. The $\mathrm{C} 106$ peak EQE $(550 \mathrm{~nm})$ is significantly reduced with the addition of AS02 on the titania surface. The EQE reduction is due to intermolecular hole transfer from the C106 dye to the AS02. The internal quantum efficiency of the control device was determined to be $88.8 \%$ for the C106 control and $72.1 \%$ with light (14\%) AS02 surface coverage.

The average excitation transfer efficiency, $\overline{\text { ETE}}$, defined as the fraction of excited NIR-ERDs that undergo energy transfer to the $\mathrm{SD}$, is described by eq $2:^{18}$

$$
\mathrm{EQE}_{\mathrm{ERD}}=\eta_{\mathrm{ABS}, \mathrm{ERD}} \cdot \mathrm{IQE} \cdot \overline{\mathrm{ETE}}
$$

where $\mathrm{EQE}_{\mathrm{ERD}}$ is the external quantum efficiency contribution caused by the NIR-ERD at $780 \mathrm{~nm}(9.4 \%), \eta_{\mathrm{ABS} \text {,ERD }}$ is the fraction of light absorbed by the NIR-ERD, and IQE is the internal quantum efficiency. The $\eta_{\mathrm{ABS} \text {, ERD }}$ was determined to be $50.8 \%$ when correcting for light losses related to reflection (4\%) and FTO light absorption (11\%) at $780 \mathrm{~nm} .{ }^{20}$ Light absorption by $\mathrm{C} 106$ at $780 \mathrm{~nm}$ was considered negligible. The estimated ETE was determined to be $26 \%$; it should be noted that the measured IQE (72.1\%) is an average value of all C106 dyes, but the IQE is most likely lower for C106 dyes that are in close proximity to ASO2, which have a higher probability of transferring holes to the AS02 before dye regeneration. Thus, the calculated $\overline{\mathrm{ETE}}$ represents the minimum bound estimate for the AS02 + C106 DSC system. AS02 is not an ideal NIR-ERD because the electron injection rate $\left(\tau_{\mathrm{inj}}<230 \mathrm{ps}\right)$ is competitive with energy transfer, which reduces the excitation transfer efficiency. For NIR-ERDs with LUMO levels above the conduction band of $\mathrm{TiO}_{2}$, an insulating ligand should be added to retard charge injection. ${ }^{21}$ If AS02 electron injection is significantly retarded, then the $\overline{\text { ETE }}$ would increase to over $70 \%$. The measured energy transfer rate $\left(k_{\mathrm{ET}}\right)$ is a combination of both Dexter and FRET energy transfer. On the basis of the $k_{\text {inj }}$ and $k_{\mathrm{nr}}$ of ASO2 and the minimum bound $\overline{\mathrm{ETE}}$ of $26 \%$, the measured rate of energy transfer $\left(k_{\mathrm{ET}}\right)$ is $>1.76 \times 10^{9} \mathrm{~s}^{-1}\left(\tau_{\mathrm{ET}}<568 \mathrm{ps}\right)$ using eq 1.

2.4. Intermolecular Hole Transfer from $\mathrm{C} 106$ to AS02. Photoinduced transient absorption (PIA) spectroscopy, shown in Figure 3, was performed on C106, ASO2 + C106, and AS02 sensitized films without the presence of the electrolyte to probe the photogenerated charge species. Steady-state PIA, which measures the change in absorption of the oxidized dye species, was chopped at a frequency of $9 \mathrm{~Hz}$ using a $470 \mathrm{~nm}$ light bias using methods previously described in the literature. ${ }^{34}$ Briefly, the C106 cation (red dash dot) bleaches at $550 \mathrm{~nm}$ and has enhanced absorption at $800 \mathrm{~nm}$, while the AS02 cation (green) bleaches at $780 \mathrm{~nm}$ and has an absorption increase at $1000 \mathrm{~nm}$. For AS02 + C106 dyed (black) films, the C106 absorbs over 80\% of the photons at the illumination wavelength $(470 \mathrm{~nm})$, but the PIA signal is dominated by the ASO2 cation, indicating intermolecular hole transfer from $\mathrm{C} 106$ to ASO2.

AS02 is an ideal dye to measure the fraction of holes from C106 dyes that transfer to NIR dyes in the cosensitized DSC system. Charge transfer between SDs in cosensitized systems has 


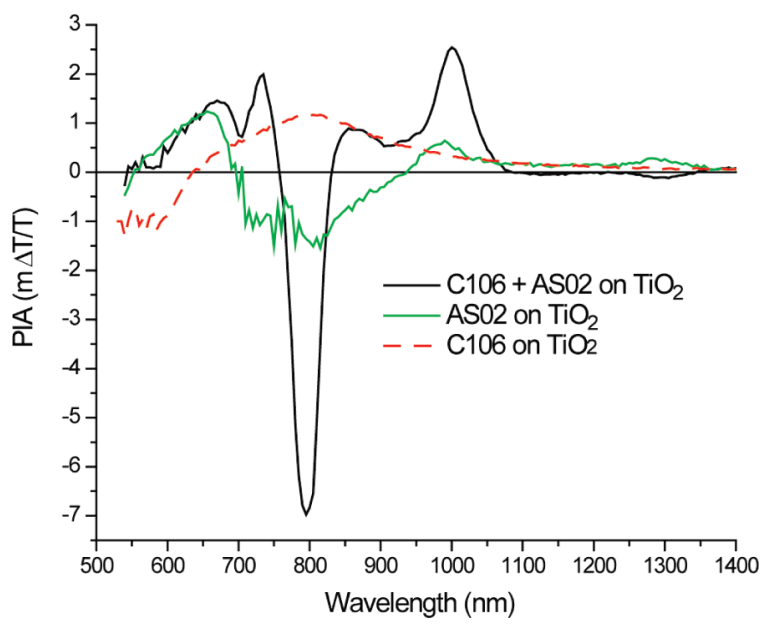

Figure 3. Photoinduced transient absorption spectra of C106 (red dash), $\mathrm{C} 106+\mathrm{ASO2}$ (black), and AS02 (green) on $\mathrm{TiO}_{2}$. PIA signals were normalized to light absorption at $470 \mathrm{~nm}$.

been previously discussed, ${ }^{10,31}$ but could not be verified nor quantified because both dyes are capable of hole regeneration. Because AS02 cannot regenerate with the electrolyte, all holes transferred to AS02 must recombine with the electrons in the $\mathrm{TiO}_{2}$ and cannot contribute to photocurrent. For this system, the fraction of holes from C106 that transfer to AS02 can be estimated on the basis of the reduction in the internal quantum efficiency of the AS02 + C106 DSC. The internal quantum efficiency is defined by eq 3 , which can be defined as the probability of hole transfer to the electrolyte, electron transfer to the titania, and the charge collection efficiency $\left(\eta_{\mathrm{CC}}\right)$. For $\mathrm{C} 106$, the electron injection rate is extremely fast relative to the nonradiative decay rate and is not expected to change with cosensitization. The $\eta_{\mathrm{CC}}$ was estimated to be $94 \%$ for C106 only but was reduced to $83 \%$ for the AS02 (56\%) + C106 (44\%) DSC system (see the Supporting Information). Further changes in the IQE will be primarily due to competition between hole transfer $\left(k_{\mathrm{HT}}\right)$ and regeneration $\left(k_{\mathrm{reg}}\right)$ of the oxidized dye by the electrolyte.

$$
\text { IQE }=\frac{k_{\text {reg }}}{k_{\mathrm{HT}}+k_{\text {reg }}+k_{\text {rec }}} \cdot \frac{k_{\text {inj }}}{k_{\text {inj }}+k_{\text {nr }}} \cdot \eta_{\mathrm{cc}}
$$

An equivalent surface concentration of ASO2 reduced the IQE from $88 \%$ for the C106 control to $47 \%$ for AS02 (56\%) + C106 (44\%) DSC (see the Supporting Information). On the basis of the IQE and $\eta_{\mathrm{CC}}$ reduction and $\mathrm{C} 106 k_{\mathrm{reg}}$ and $k_{\mathrm{rec}}$ rates, the effective hole transfer lifetime, $\tau_{\mathrm{HT}}$, is $5.4 \mu \mathrm{s}$. It should be noted that this is an averaged rate over all C106 dyes cosensitized on the $\mathrm{TiO}_{2}$ surface; the intermolecular hole transfer rate may significantly vary depending on how $\mathrm{C} 106$ and AS02 pack with one another on the surface. While the IQE reduction caused by ASO2 is an extreme case, regeneration rates can be slower for organic dyes, and NIR dyes in particular will likely have a lower driving force for hole regeneration. ${ }^{35,36}$ The $k_{\mathrm{HT}}$ indicates that $>40 \%$ holes can be transferred from $\mathrm{C} 106$ dyes near AS02. Intermolecular hole migration to NIR dyes has important implications for $V_{\text {oc }}$.

2.5. Negative Effects of Intermolecular Hole Transfer in Cosensitized Dye-Sensitized Solar Cells. A $80 \mathrm{mV}$ drop in $V_{\text {oc }}$ was observed for the cosensitized AS02 (14\%) + C106 (86\%) DSC $\left(V_{\mathrm{oc}}=650 \mathrm{mV}\right)$ system relative to the C106 control DSC $\left(V_{\mathrm{oc}}=730 \mathrm{mV}\right)$ (see the Supporting Information). Because the

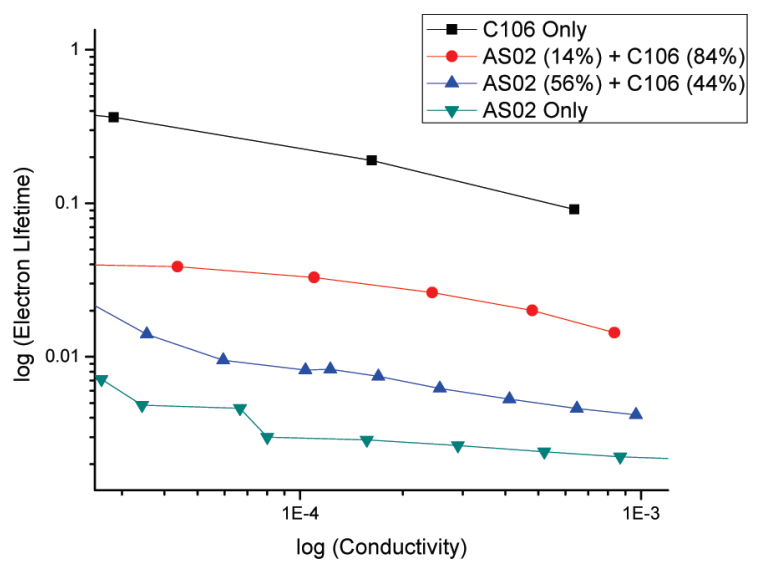

Figure 4. Electron lifetime versus conductivity for DSC systems with various concentrations of $\mathrm{ASO} 2$ and $\mathrm{C} 106$ on $\mathrm{TiO}_{2}$ in the dark.

$V_{\text {oc }}$ is also affected by the reduction in the photocurrent density, the electron lifetime was studied to determine the effects of intermolecular hole migration on the recombination. The electron lifetime was measured using electrochemical impedance spectroscopy for various fractional AS02/C106 surface concentrations to better understand the change in $V_{\text {oc }}$. Impedance measurements were performed with an Autolab PGSTAT30 (EcoChemie B.V., Utrecht, Netherlands) over a frequency range from $1 \mathrm{MHz}$ to $0.1 \mathrm{~Hz}$ at bias potentials between -0.2 and $-0.8 \mathrm{~V}$ (with a $10 \mathrm{mV}$ sinusoidal AC perturbation); all measurements were done at $20^{\circ} \mathrm{C}$ and in the dark. The resulting impedance spectra were analyzed with ZView software (Scribner Associate Inc.) on the basis of the two-channel transmission line model. ${ }^{37,38}$ The electron lifetimes of various AS02 $+\mathrm{C} 106$ cosensitized DSC systems are plotted against conductivity of the $\mathrm{TiO}_{2}$ and are shown in Figure 4. C106-only DSCs have an electron lifetime of $500 \mathrm{~ms}$, while AS02-only DSCs have an electron lifetime of $2 \mathrm{~ms}$ near open-circuit voltage conditions. If the dyes do not electronically interact in the cosensitized DSC system, then one might expect the electron decay rate to be the weighted average of the individual dye systems. However, in the AS02 (14\%) + C106 (86\%) cosensitized DSC system, the electron lifetime is $50 \mathrm{~ms}$, which is nearly 3 times lower than the weighted lifetime of $140 \mathrm{~ms}$. The disproportionate change in electron lifetime may be caused by hole transfer from the $\mathrm{C} 106$ to AS02. The $V_{\mathrm{oc}}$ change is not related to a reduction in the overall dye loading on the $\mathrm{TiO}_{2}$, which actually increases during cosensitization (see the Supporting Information). It should be noted that recombination between electrons in the titania and the $\mathrm{I}_{3}{ }^{-}$electrolyte is generally considered to be the $V_{\text {oc }}$-determining recombination mechanism when using Ru-based metal complex dyes, which have relatively fast regeneration rates. ${ }^{13,39}$ However, recombination from electrons in $\mathrm{TiO}_{2}$ to oxidized dye species may become the critical mechanism for NIR dyes whose ground-state redox potentials are less favorable for regeneration. A complete study of the recombination kinetics of fully functioning NIR-SD is required to determine which recombination mechanism plays a dominant role under $V_{\text {oc }}$ conditions for cosensitized DSC systems.

\section{CONCLUSION}

This study demonstrates the need to refine design rules for NIR-SDs and NIR-ERDs. NIR-SDs should have sufficient 
LUMO and HOMO levels for charge injection and a high molar extinction coefficient $\left(>100000 \mathrm{M}^{-1} \mathrm{~cm}^{-1}\right)$. Planar NIR-SDs that pack well with metal ligand SDs may lose substantial $V_{\text {oc }}$ due to intermolecular hole transfer, negating the potential power conversion efficiency gain with high $V_{\text {oc }}$ losses. NIR-SDs should be physically separated from the metal complex SD either via long alkyl side chains or via selective positioning ${ }^{9,40}$ to prevent intermolecular hole transfer to maintain high open-circuit voltage.

NIR-ERDs do not require precise LUMO level alignment and short conjugated ligands for rapid electron charge injection. However, NIR-ERDs must intimately mix with metal complex sensitizing dyes to efficiently transfer energy and must therefore have a HOMO level below the iodide potential to regenerate with the electrolyte. Ideally, NIR-ERDs should be designed with an insulating ligand that is long enough to prevent electron transfer $^{21}$ and lower recombination, but short enough to enable close range interactions with the SD. Optimized NIR-ERD for use in conjunction with bipyridyl ruthenium complexes should have peak absorption between 720 and $790 \mathrm{~nm}$ and peak emission between 730 and $800 \mathrm{~nm}$. Dyes with lower band gaps (i.e., dyes with an emission peak $>820 \mathrm{~nm}$ ) would most likely not work as NIR-ERDs with ruthenium-based SDs. The ability to both sensitize and transfer energy from NIR-ERDs to metal complex sensitizing dyes allows us to expand the light harvesting out to $800 \mathrm{~nm}$, which has the potential to improve highly efficient DSCs in the future if the negative effects of intermolecular hole transfer can be mitigated.

\section{ASSOCIATED CONTENT}

S Supporting Information. Synthetic and experimental details. This material is available free of charge via the Internet at http://pubs.acs.org.

\section{AUTHOR INFORMATION}

\section{Corresponding Author}

mmcgehee@stanford.edu

\section{ACKNOWLEDGMENT}

B.E.H. would like to thank Jun-Ho Yum for preparing the $\mathrm{TiO}_{2}$ films, Jason Bloking for PESA measurements, and Eric Hoke for helpful discussions. This work was primarily supported by the Office of Naval Research contract no. N00014-08-1-1163. P.W. thanks the Key Scientific Program (No. 2007CB936700).

\section{REFERENCES}

(1) O’Regan, B.; Grätzel, M. Nature 1991, 353, 737.

(2) Hamann, T. W.; Jensen, R. A.; Martinson, A. B. F.; Ryswyk, H. V.; Hupp, J. T. Energy Environ. Sci. 2008, 1, 66.

(3) Snaith, H. J.; Schmidt-Mende, L. Adv. Mater. 2007, 19, 3187.

(4) Hagfeldt, A.; Gratzel, M. Acc. Chem. Res. 2000, 33, 269.

(5) Cao, Y.; Bai, Y.; Yu, Q.; Cheng, Y.; Liu, S.; Shi, D.; Gao, F.; Wang, P. J. Phys. Chem. C 2009, 113, 6290.

(6) Nazeeruddin, M. K.; De Angelis, F.; Fantacci, S.; Selloni, A.; Viscardi, G.; Liska, P.; Ito, S.; Takeru, B.; Grätzel, M. J. Am. Chem. Soc. 2005, 127, 16835 .

(7) Cid, J.-J.; Yum, J.-H.; Jang, S.-R.; Nazeeruddin, M. K.; MartínezFerrero, E.; Palomares, E.; Ko, J.; Grätzel, M.; Torres, T. Angew. Chem. 2007, 119, 8510.

(8) Chen, Y.; Zeng, Z.; Li, C.; Wang, W.; Wang, X.; Zhang, B. New J. Chem. 2006, 29, 773.
(9) Choi, H.; Kim, S.; Kang, S. O.; Ko, J.; Kang, M.-S.; Clifford, J. N.; Forneli, A.; Palomares, E.; Nazeeruddin, M. K.; Grätzel, M. Angew. Chem. 2008, 120, 8383.

(10) Ehret, A.; Stuhl, L.; Spitler, M. T. J. Phys. Chem. B 2001, 105,9960

(11) Ono, T.; Yamaguchi, T.; Arakawa, H. Sol. Energy Mater. Sol. Cells 2009, 93, 831.

(12) Macor, L.; Fungo, F.; Tempesti, T.; Durantini, E. N.; Otero, L.; Barea, E. M.; Fabregat-Santiago, F.; Bisquert, J. Energy Environ. Sci. 2009, 2, 529.

(13) Peter, L. M. Phys. Chem. Chem. Phys. 2007, 9, 2630.

(14) Miyashita, M.; Sunahara, K.; Nishikawa, T.; Uemura, Y.; Koumura, N.; Hara, K.; Mori, A.; Abe, T.; Suzuki, E.; Mori, S. J. Am. Chem. Soc. 2008, 130, 17874.

(15) Hu, X.; Schulten, K. Phys. Today 1997, 50, 28.

(16) Förster, T. Discuss. Faraday Soc. 1959, $27,7$.

(17) Dexter, D. L. J. Chem. Phys. 1953, 21, 836.

(18) Hardin, B. E.; Hoke, E. T.; Armstrong, P. B.; Yum, J.-H.; Comte, P.; Torres, T.; Frechet, J. M. J.; Nazeeruddin, M. K.; Grätzel, M.; McGehee, M. D. Nat. Photonics 2009, 3, 406.

(19) Siegers, C.; Hohl-Ebinger, J.; Zimmermann, B.; Würfel, U.; Mülhaupt, R.; Hinsch, A.; Haag, R. ChemPhysChem 2007, 8, 1548.

(20) Hardin, B. E.; Yum, J.-H.; Hoke, E. T.; Jun, Y. C.; Péchy, P.; Torres, T.; Brongersma, M. L.; Nazeeruddin, M. K.; Grätzel, M.; McGehee, M. D. Nano Lett. 2010, 10, 3077.

(21) Siegers, C.; Würfel, U.; Zistler, M.; Gores, H.; Hohl-Ebinger, J.; Hinsch, A.; Haag, R. ChemPhysChem 2008, 9, 793.

(22) Shankar, K.; Feng, X.; Grimes, C. A. ACS Nano 2009, 3, 788.

(23) Hoke, E. T.; Hardin, B. E.; McGehee, M. D. Opt. Express 2010, $18,3893$.

(24) Nazeeruddin, M. K.; Kay, A.; Rodicio, I.; Humphry-Baker, R.; Mueller, E.; Liska, P.; Vlachopoulos, N.; Graetzel, M. J. Am. Chem. Soc. 1993, 115, 6382.

(25) Ishii, K.; Kobayashi, N. The Porphyrin Handbook; Elsevier Science: New York, 2003; Vol. 16.

(26) Li, X.; Long, N. J.; Clifford, J. N.; Campbell, C.; Durrant, J. New J. Chem. 2002, 26, 1076.

(27) Lakowicz, J. R. Principles of Fluorescence Spectroscopy, 2nd ed.; Plenum: New York, 1999.

(28) Farzad, F.; Thompson, D. W.; Kelly, C. A.; Meyer, G. J. J. Am. Chem. Soc. 1999, 121, 5577.

(29) Higgins, G. T.; Bergeron, B. V.; Hasselmann, G. M.; Farzad, F.; Meyer, G. J. J. Phys. Chem. B 2006, 110, 2598.

(30) Kuang, D.; Ito, S.; Wenger, B.; Klein, C.; Moser, J.-E.; Humphry-Baker, R.; Zakeeruddin, S. M.; Grätzel, M. J. Am. Chem. Soc. 2006, 128, 4146.

(31) Sayama, K.; Tsukagoshi, S.; Mori, T.; Hara, K.; Ohga, Y.; Shinpou, A.; Abe, Y.; Suga, S.; Arakawa, H. Sol. Energy Mater. Sol. Cells 2003, 80, 47.

(32) Sommeling, P. M.; O’Regan, B. C.; Haswell, R. R.; Smit, H. J. P.; Bakker, N. J.; Smits, J. J. T.; Kroon, J. M.; van Roosmalen, J. A. M. J. Phys. Chem. B 2006, 110, 19191.

(33) Ito, S.; Murakami, T. N.; Comte, P.; Liska, P.; Grätzel, C.; Nazeeruddin, M. K.; Grätzel, M. Thin Solid Films 2008, 516, 4613.

(34) Snaith, H.J.; Humphry-Baker, R.; Chen, P.; Cesar, I.; Zakeeruddin, S. M.; Gratzel, M. Nanotechnology 2008, 19, 424003.

(35) Tatay, S.; Haque, S. A.; O’Regan, B.; Durrant, J. R.; Verhees, W. J. H.; Kroon, J. M.; Vidal-Ferran, A.; Gavina, P.; Palomares, E. J. Mater. Chem. 2007, 17, 3037.

(36) Ardo, S.; Meyer, G. J. Chem. Soc. Rev. 2009, 38, 115.

(37) Fabregat-Santiago, F.; Bisquert, J.; Garcia-Belmonte, G.; Boschloo, G.; Hagfeldt, A. Sol. Energy Mater. Sol. Cells 2005, 87, 117.

(38) Fabregat-Santiago, F.; Bisquert, J.; Palomares, E.; Otero, L.; Kuang, D.; Zakeeruddin, S. M.; Grätzel, M. J. Phys. Chem. C 2007, 111,6550

(39) Pelet, S.; Moser, J.-E.; Gratzel, M. J. Phys. Chem. B 2000, 104, 1791.

(40) Lee, K.; Park, S. W.; Ko, M. J.; Kim, K.; Park, N.-G. Nat. Mater. 2009, 8, 665 . 